\title{
GLP-1 Val8: A biased GLP-1R agonist with altered binding kinetics and impaired release of pancreatic hormones in rats
}

Wijnand J. C. van der Velden ${ }^{1}$, Florent X. Smit ${ }^{1}$, Charlotte B. Christiansen ${ }^{2,3}$, Thor C. Møller ${ }^{4}$, Gertrud M. Hjortø1, Olav Larsen${ }^{1}$, Sine P. Schiellerup ${ }^{1}$, Hans BräunerOsborne $^{4}$, Jens J. Holst ${ }^{2,3}$, Bolette Hartmann ${ }^{2,3}$, Thomas M. Frimurer ${ }^{5}$, Mette M. Rosenkilde ${ }^{1 *}$

1 Laboratory for Molecular Pharmacology, Department of Biomedical Sciences, Faculty of Health and Medical Sciences, University of Copenhagen, Copenhagen, Denmark

${ }^{2}$ Department of Biomedical Sciences, Faculty of Health and Medical Sciences, University of Copenhagen, Copenhagen, Denmark

${ }^{3}$ Novo Nordisk Foundation Center for Basic Metabolic Research, Faculty of Health and Medical Sciences University of Copenhagen, Copenhagen, Denmark

${ }^{4}$ Department of Drug Design and Pharmacology, Faculty of Health and Medical Sciences, University of Copenhagen, Copenhagen, Denmark

${ }^{5}$ Section for Metabolic Receptology, Novo Nordisk Foundation Center for Basic Metabolic Research, Faculty of Health and Medical Sciences, University of Copenhagen, Copenhagen, Denmark

*corresponding author. Email: rosenkilde@sund.ku.dk 
Table of Contents

Table S-1 | Relaxation of the in-silico homology model.......................................

Table S-2 | cAMP accumulation in rat and human GLP-1R ................................

Figure S-1| Secondary structure characterization through circular dichroism. 4 Figure S-2 | Internalization of GLP-1R by GLP-1 and GLP-1 Val8. .....................5

Figure S-3 | Molecular dynamic simulations of GLP-1R (PDB: 5VAl) with GLP-1

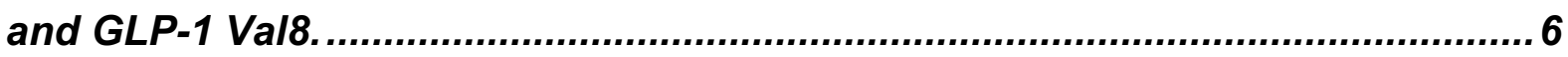

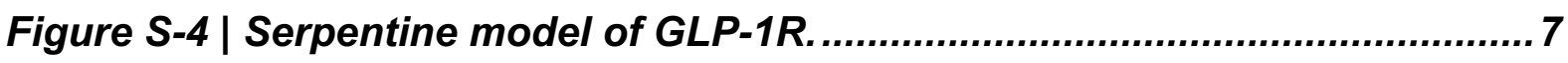

Figure S-5 | Dose-response curves of cAMP production on 27 alanine

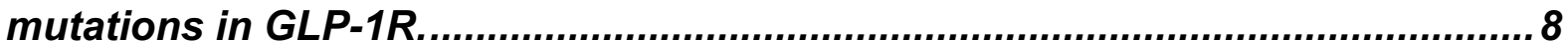


Table S-1 | Pharmacology in rat and human GLP-1R

\begin{tabular}{|l|l|l|l|l|l|l|}
\hline & \multicolumn{3}{|c|}{ cAMP accumulation } & \multicolumn{3}{c|}{$\beta$-arrestin 2 recruitment } \\
\hline & \multicolumn{2}{|c|}{ rat GLP-1R $(\mathrm{n}=3)$} & \multicolumn{2}{c|}{ human GLP-1R $(\mathrm{n}=3)$} & \multicolumn{2}{c|}{ rat GLP-1R $(\mathrm{n}=3)$} \\
\hline Ligand & $\mathrm{pEC} 50$ & $\mathrm{E}_{\max }(\%)$ & $\mathrm{pEC} 50$ & $\mathrm{E}_{\max }(\%)$ & $\mathrm{pEC} \mathrm{C}_{50}$ & $\mathrm{E}_{\max }(\%)$ \\
\hline GLP-1 & $10.2 \pm 0.1$ & $100 \pm 0.0$ & $10.2 \pm 0.1$ & $100 \pm 0.0$ & $8.2 \pm 0.5$ & $100 \pm 0.0$ \\
\hline GLP-1 Val8 & $10.1 \pm 0.2$ & $100 \pm 11.2$ & $9.7 \pm 0.1$ & $96 \pm 3.6$ & $8.0 \pm 0.1$ & $49 \pm 5.4^{*}$ \\
\hline
\end{tabular}

Human GLP-1R was tested in parallel with the rat GLP-1R. All data were fitted with the three-parameter logistic curve to obtain $\mathrm{pEC}_{50}$ and $\mathrm{E}_{\max } \mathrm{pEC_{50 }}$ represents the negative logarithm of agonist concentration in molar that produces half the maximal response. $E_{\max }$ is characterized as the maximal response normalized to the GLP-1 response. Data represent the mean \pm s.e.m. of $\mathrm{n}$ independent experiments performed duplicate. Statistical significance was assessed using a two-tailed paired $t$-test for $E_{\max }$ $\left({ }^{*} P<0.05\right.$; as compared to GLP-1 response).

\section{Table S-2 | Relaxation of the in-silico homology model}

\begin{tabular}{|c|c|c|c|c|c|}
\hline Stage & Ensemble & Lipids (positional) & Lipids (dihedral) & Protein (bb) & Protein (sc) \\
\hline Minimization & St. descent & 1000 & 1000 & 4000 & 2000 \\
\hline Stage 1 & NVT & 1000 & 1000 & 4000 & 2000 \\
\hline Stage 2 & NVT & 1000 & 400 & 2000 & 1000 \\
\hline Stage 3 & NPT & 400 & 200 & 1000 & 500 \\
\hline Stage 4 & NPT & 200 & 200 & 500 & 200 \\
\hline Stage 5 & NPT & 40 & 100 & 200 & 50 \\
\hline Stage 6 & NPT & 0 & 0 & 50 & 0 \\
\hline Production run & NPT & 0 & 0 & 0 & 0 \\
\hline
\end{tabular}

Summary of the ensemble and restraints applied in the six-stage relaxation procedure of the molecular dynamics simulations of the GLP-1R. Units are $\mathrm{In} \mathrm{kJ} / \mathrm{mol}$. bb is backbone and sc is side chain. 

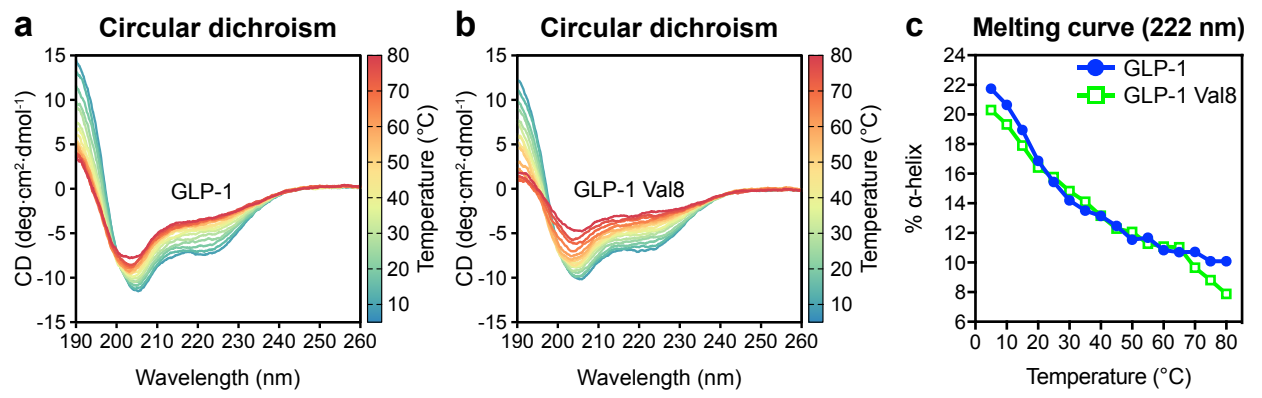

Figure S-1 | Secondary structure characterization through circular dichroism.

Measured mean residue molar ellipticity $\left([\theta]_{\mathrm{mr}}\right.$ ) for both GLP-1 (a) and GLP-1 Val8 (b) for the temperature range 5 to $80^{\circ} \mathrm{C}$ (colorbar). A clearly distinguishable double valley appeared, indicating that both GLP-1 and GLP-1 Val8 display an a-helical nature ${ }^{84}$. In addition, we observed a loss of this distinct absorbance upon heating of the sample, indicating the transition from helical to a disordered state. (c) The corresponding melting curve obtained by plotting the $[\theta]_{\mathrm{mr}}$ at $222 \mathrm{~nm}$ as a function of temperature. This value gives an indirect measure of the peptide's helical propensity, which can be estimated using equation (6). 


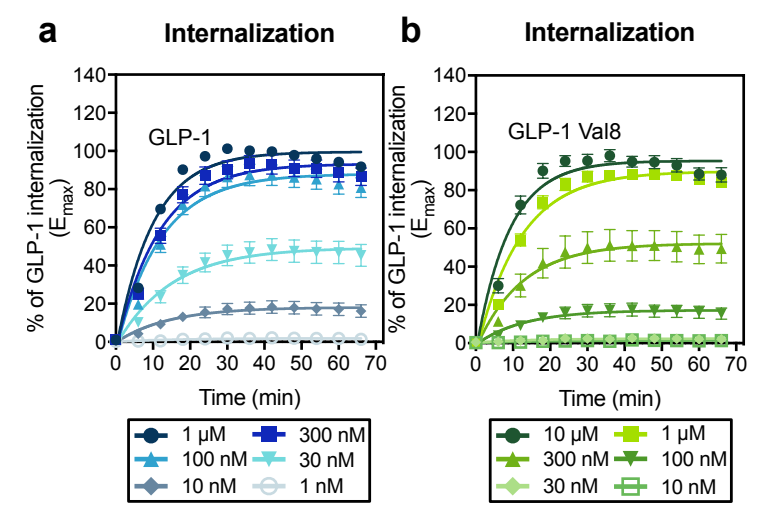

Figure S-2 | Internalization of GLP-1R by GLP-1 and GLP-1 Val8.

TR-FRET internalization (a) for GLP-1 and (b) GLP-1 Val8 in HEK 293A cells expressing SNAP-tagged human GLP-1R. Data represent the mean \pm s.e.m. of 6 independent experiments performed in duplicate and triplicate. 

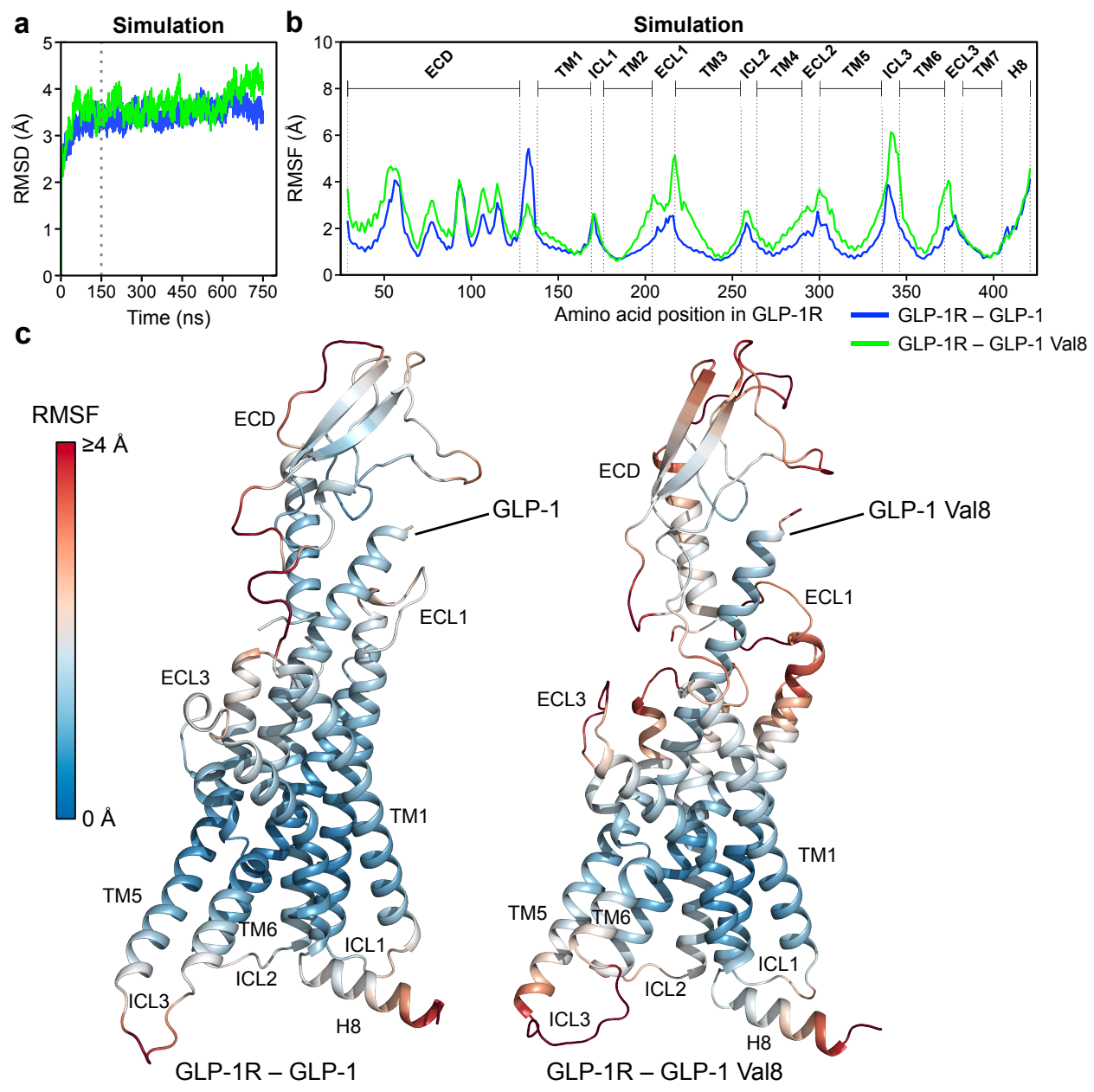

Figure S-3 | Molecular dynamic simulations of GLP-1R (PDB: 5VAI) with GLP-1 and GLP-1 Val8.

Different parameters to determine the stability and mobility of the simulated receptor. (a) Root-mean-square deviation (RMSD) of GLP-1R in complex with GLP-1 or GLP-1 Val8, relative to the starting structure. Root-mean-square fluctuation (RMSF) of regions in GLP-1R when bound to GLP-1 or GLP-1 Val8 displayed as graph (b) and visualized in structure of GLP-1R (c). 


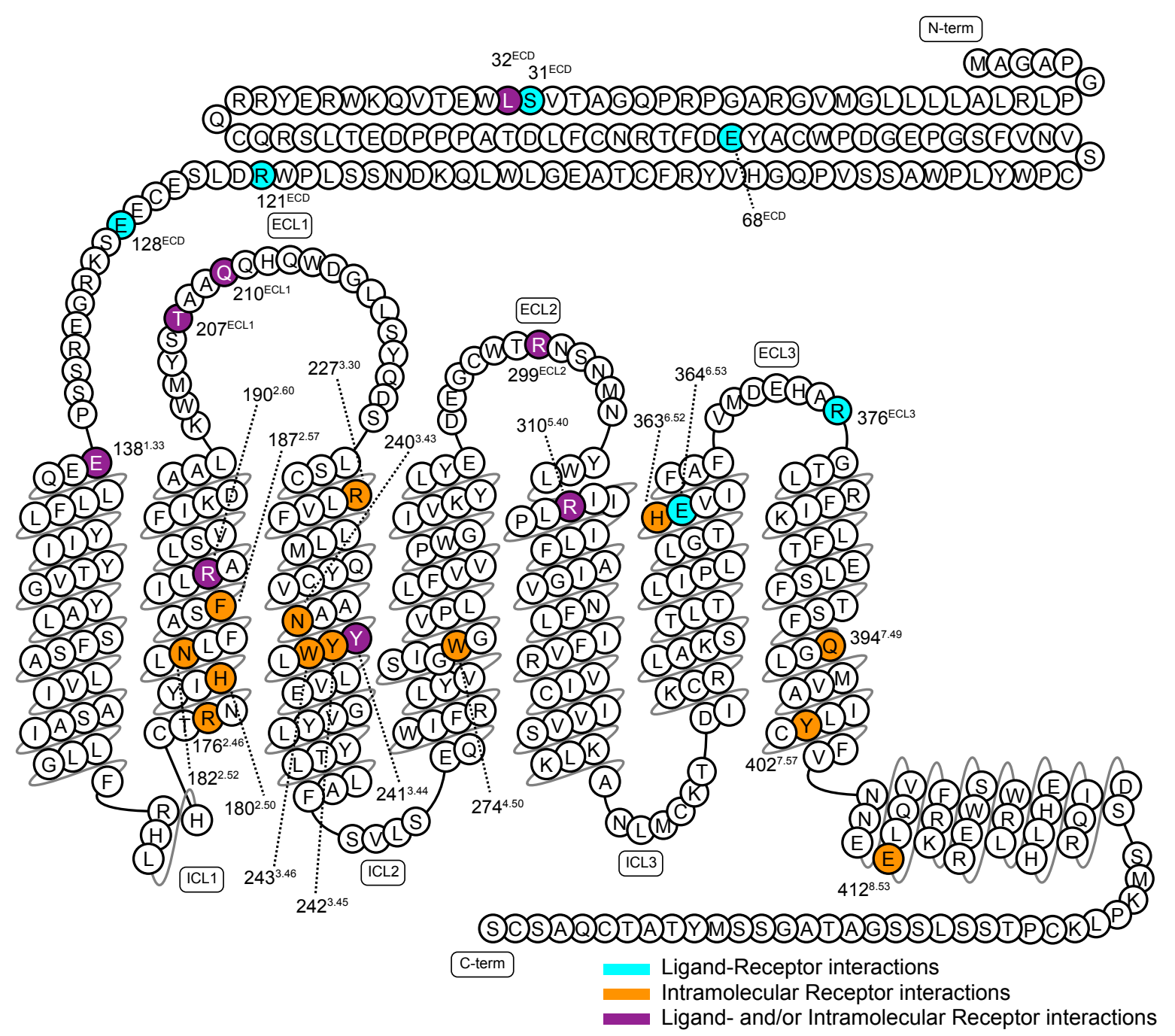

Figure S-4 | Serpentine model of GLP-1R.

The 27 substitutions to alanine are colored based on their interaction pattern: Ligandreceptor, Intramolecular receptor and Ligand and/or intramolecular. 

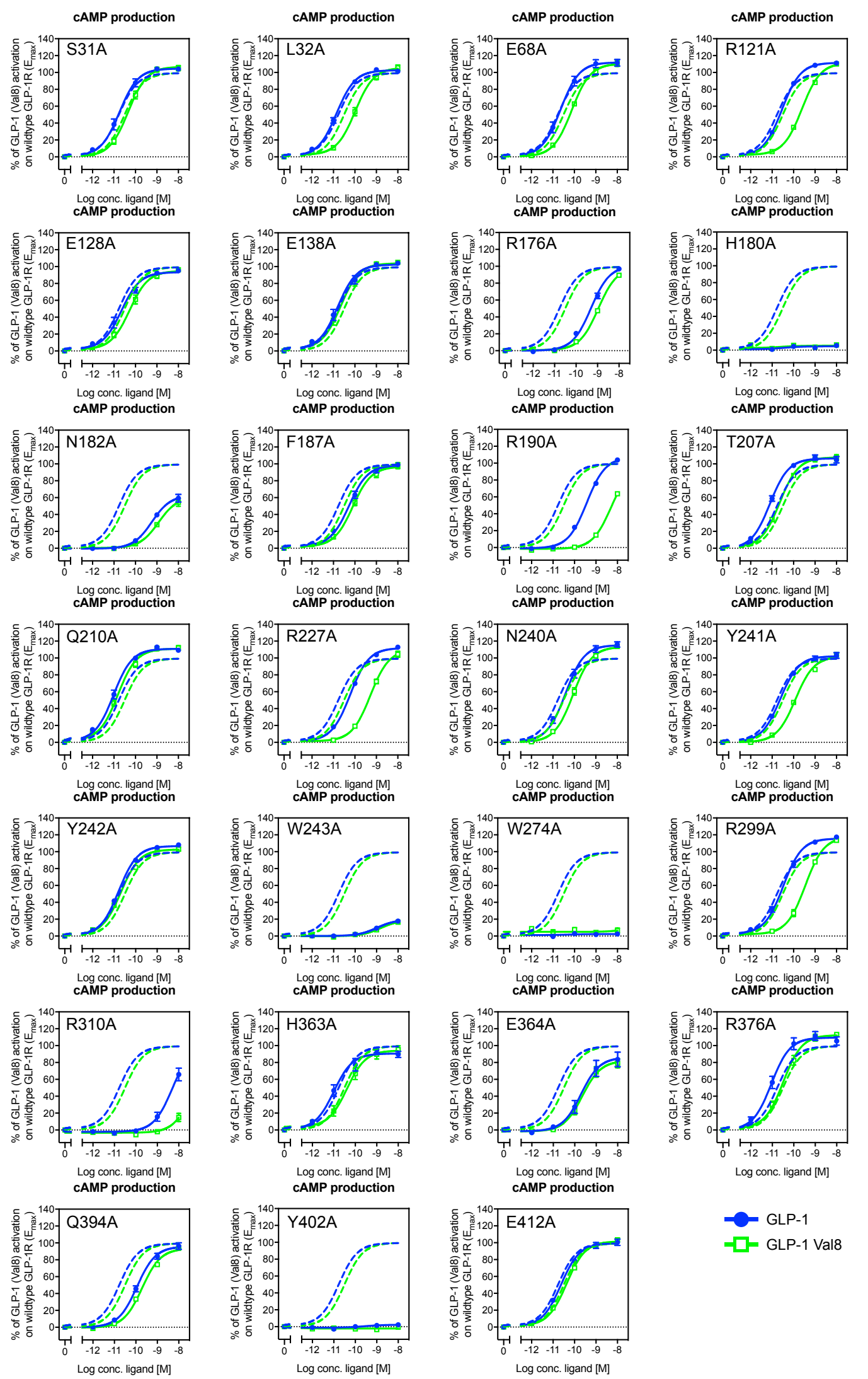

Figure S-5 | Dose-response curves of cAMP production on 27 alanine mutations in GLP-1R. 
Data were normalized to GLP-1R WT for both GLP-1 and GLP-1 Val8. Wildtype curves are presented as dashed lines. Data represent the mean \pm s.e.m. of 18 independent experiments performed in duplicate on WT, and 3-4 on the mutants. 